\title{
Viewpoint: Sample adequacy for point analysis depends on the objectives
}

\author{
MELCHOR ORTIZ AND MICHAEL H. AMES
}

\begin{abstract}
Authors are professor and assistant professor, Department of Experimental Statistics, New Mexico State University, Las Cruces 88003.
\end{abstract}

Hofmann and Ries (1990) address the question of determining the required sample size in point analysis of ground cover for proving successful reclamation of a mining site according to government regulations from a sample of 10 -pin point frames. The formulas presented are different forms of equation (1)

$$
\hat{\mathbf{n}}=(\mathbf{t s})^{2} /(.10 \mathrm{p})^{2}
$$

where $.10 \mathrm{p}$ is $10 \%$ of the reference cover. Hofmann and Ries analyze the behavior of this formula on actual data sets, criticize it, and suggest an alternative that seems to reduce the sample size by a (suspiciously) considerable amount. This short paper is at best inaccurate and at worst seriously misleading. Their critique compares the wrong numbers and we suggest that the authors are using the right formula but for the wrong problem. A further confounding issue is that the federal rules and regulations do not give a clear path to resolving the question of how to establish the proper sample size.

The idea that increasing sample size should produce more 'consistent' means and variances is one that merits close attention. It is well known that $\bar{x}$ is an unbiased estimate of $\mu$ and that $\mathrm{s}^{2}$ is an unbiased estimate of $\sigma^{2}$. That is, on average the mean of the $\bar{x}$ 's is the population mean regardless of the sample size. However, variation of $\bar{x}$ 's about the population mean decreases with increasing sample size. This is expressed in the formula that the variance of a sample mean is $\sigma^{2} / \mathbf{n}$. The population mean and variance (that is, the mean and variance of the $x$ 's) do not change with increasing sample size, but the variance of the sample mean and sample variance do decrease. Note that the variances presented in Table 1 of Hofmann and Ries's paper are not variances of $\bar{x}$ 's but rather estimates of the population variances. The data in Table 1 do not address the issue of the consistency of the $\bar{x}$ 's. It is inaccurate to look at the means presented and decide that increasing sample size did not produce more 'consistent' means.

The authors correctly point out that equation (1) is inappropriate when the original population of values is not normal. It is sufficient, however, that the $\bar{x}$ 's be normally distributed. This is usually assured by the Central Limit Theorem with large sample sizes.

Equation (2), which the authors propose as a better solution, has

$$
\hat{\mathbf{n}}=\left(\mathrm{t}^{2} \mathrm{pq}\right) / .10^{2}
$$

a couple of drawbacks. Note especially that the denominator has been set to be exactly 0.10 . This is quite different from the required $10 \%$ of the mean as before! The proper denominator is $0.10 \mathrm{p}$ so that

$$
\hat{\mathbf{n}}=\left(\mathrm{t}^{2} \mathrm{pq}\right) /(0.10 \mathrm{p})^{2} .
$$

Using this change, the first 4 values of $\hat{n}$ in Table 1 should be about $570,430,450$, and 480 instead of $33,35,34$, and 33 .

Secondly, since the readings for pins on a frame are not inde- pendent, the binomial distribution is incorrect anyway; i.e., does not correctly describe the distribution of the number of pins hitting plant material.

These are minor quibbles. The major problem with the above approach is that it is the correct solution but to the wrong problem. The federal regulation states: "the ground cover and productivity of the revegetated area shall be considered equal [to the reference area] if they are at least $90 \%$ of the ground cover and productivity of the reference area with $90 \%$ statistical confidence." This must mean that, after taking a sample, if a one-sided $t$-test can reject the hypothesis that the cover of the reclaimed site is less than or equal to $90 \%$ of the reference site with a Type I error rate of $10 \%$, then the site is considered successfully reclaimed. Consider the following example: if the cover for the reference area is $25 \%$, we want to prove that the cover for the reclaimed site is at least $22.5 \%$. Using the sample size calculated from the equations in the Hofmann and Ries paper we can be $90 \%$ confident that we can estimate the cover of the reclaimed site to within $2.5 \%$ - whether this cover is actually $10 \%$ or $50 \%$, which does not address the issue of whether the mean at the reclaimed site is or is not greater than $22.5 \%$. Even if the reclaimed site has the same cover as the reference site, the hypothesis we want to reject will only be rejected about half the time using this sample size. The question that should be asked is: how large a sample is necessary to prove with a $10 \%$ Type I error rate that the mean is at least $90 \%$ of the reference mean? (The next question is: how confident do you want to be that the sample size will be sufficient to prove the mean is at least this amount?) Unfortunately, this question cannot be answered because it depends on the true value of the unknown mean cover and unknown variance. Let's assume that the reclaimed site's cover is greater than .9p. If it is barely greater, then a larger sample size would be necessary to prove the mean is above .9p than if it were much greater than .9p. If the reclaimed site's cover were less than $.9 p$, then there is no sample size that will assure us that we can prove the mean is greater than $.9 p$.

This problem is not solved with the methods given in the Hofmann and Ries paper.

\section{Literature Cited}

Hofmann, L., and R.E. Ries. 1990. An evaluation of sample adequacy for point analysis of ground cover. J. Range Manage. 43:545-549. 